A - Research concept and design

B - Collection and/or assembly of data

C - Data analysis and interpretation

D - Writing the article

E - Critical revision of the article

F - Final approval of article

Received: 2021-03-26 Accepted: 2021-06-24 Published: 2021-07-02

\section{Effect of vestibular rehabilitation therapy on spatio-temporal gait parameters in elderly patients with post-stroke hemineglect}

\author{
Mohammed Youssef Elhamrawy ${ }^{1,2}$ (iD, Sherin Mohamed ${ }^{3}$ (D), \\ Wafik Bahnasy ${ }^{4}$ iD, Mohamed Yasser Saif ${ }^{5}$ iD, Amr Elkholy6 iD, \\ Mohamed Said ${ }^{1}$
}

${ }^{1}$ Department of Physical Therapy for Elderly, National Institute for Longevity Elderly Sciences (NILES), Beni-Suef University, Beni-Suef, Egypt ${ }^{2}$ Kafr El Zayat General Hospital, Ministry of Health and Population, Al Gharbiyah, Egypt

${ }^{3}$ Departement of Physical Therapy for Internal Medicine, Faculty of Physical Therapy, Beni-suef University, Egypt

${ }^{4}$ Department of Neuropsychiatry, Faculty of Medicine, Tanta University, Egypt

${ }^{5}$ Departement of Ophthalmology, Faculty of Medicine, Beni Suef University, Egypt

${ }^{6}$ Departement of Computer and Control Engineering, Faculty of Engineering, Tanta University, Egypt

*Correspondence: Mohammed Youssef Elhamrawy; Department of Physical Therapy for Elderly, National Institute for Longevity Elderly Sciences (NILES), BeniSuef University, Beni-Suef,Kafr El Zayat General Hospital, Ministry of Health and

Population, Al Gharbiyah, Egypt; email: dr_melhamrawy@yahoo.com

\title{
Abstract
}

Introduction: Hemineglect is a lack of awareness for the contralesional space. It is a complex neurologic condition to rehabilitate. The study was designed to investigate the effects of vestibular rehabilitation therapy (VRT) on spatio-temporal gait parameters in hemineglect.

Material and methods: Thirty-two hemineglect patients were randomly assigned to the experimental and the control group. The experimental group received 60 minutes of training (4 days/week) for the first four weeks (40 minutes traditional physiotherapy and 20 minutes VRT) and then completed the following four weeks with 60 minutes of only traditional physiotherapy. For eight weeks, the control group completed 60 minutes of the traditional program four days a week. The Microsoft Kinect V2 was used to measure spatio-temporal gait parameters. Patients were assessed at baseline, four and eight weeks post-intervention.

Results: After four and eight weeks of intervention, the experimental group demonstrated a significant improvement in walking speed $(\mathrm{P}=0.0002, \mathrm{~d}=12.38$ and $\mathrm{P}=0.001, \mathrm{~d}=13.69$, respectively), cadence $(\mathrm{P}=0.0003, \mathrm{~d}=3.88$, and $\mathrm{P}=0.0003, \mathrm{~d}=5.19$, respectively), paretic step length $(\mathrm{P}=0.0001, \mathrm{~d}=2.53$, and $\mathrm{P}=0.001, \mathrm{~d}=3.84$, respectively $)$, and non-paretic step length $(\mathrm{P}=0.0119, \mathrm{~d}=2.06$, and $\mathrm{P}=0.0044, \mathrm{~d}=2.31$, respectively). There were no significant differences in the control group in any of the spatio-temporal gait parameters.

Conclusions: VRT improves the spatio-temporal gait parameters in hemineglect, allowing patients to walk more securely and navigate more easily during walking. VRT might improve the patients' postural control, weight distribution, and orientation during walking. This improvement was maintained following training, and additional improvements in spatio-temporal gait parameters were observed compared to baseline.

Keywords: gait, neglect, Vestibular Rehabilitation Therapy

This is an Open Access journal, all articles are distributed under the terms of the Creative Commons Attribution-NonCommercial-ShareAlike 4.0 International (CC BY-NC-SA 4.0). License (http://creativecommons. org/licenses/by-nc-sa/4.0/). 


\section{Introduction}

Hemineglect is a complex disabling disorder commonly following a right-sided hemispheric stroke [1]. It is commonly caused by large strokes in the territory of the middle cerebral, particularly those affecting the temporo-parieto-occipital area [2]. Patients with behavioral abnormalities, paralysis neglect, or primary lesion to the visual geniculostriate pathways must be excluded from the diagnosis [3]. It can be influenced by various pathological processes, including neurodegenerative disease, trauma, and neoplasia [4].

Impairment of this condition affects patients' ability to perform daily activities because they are unwilling to report, respond, or orient to meaningful stimuli or even objects located to their contralateral lesion side [5]. Neglect was associated with longer hospitalization, poor functional outcomes, dependency for assisted care, and reduction in psychological well-being compared to stroke survivors without neglect. Therefore, it is a prognostic indicator of poorer functional independence after stroke [6].

Patients undergoing rehabilitation for neglect demonstrate related attentional-perceptual deficits that affect their navigation abilities $[7,8]$. The neglect phenomenon impairs heading control, resulting in a deviation in the walking trajectory [9]. Patients hardly navigate safely, walking with lateral deviation trajectory that deviates rightward from the midline, discarding all lines on the left side of the paper, copying only half of the object, and denying difficulties with perception or control of movement, are unaware that they are experiencing these symptoms. Patients usually have a decreased awareness of their deficits [7].

Post-stroke impairments in locomotion are frequently observed, including reduced speed and increased gait inter-limb asymmetry [10]. Patients post-stroke usually suffer from weight-bearing asymmetry and postural sway. The risk of post-stroke falls increased with increasing older age and impaired postural control $[11,12]$.

Walking safely requires both executive and cognitive control to be intact. Gait deficits increase the risk of falls and injuries, and reduction in independence. Speed, cadence, step length, stride length, and base width are all main measures of gait variables [13]. Because a high proportion of stroke survivors fall while walking (40-90\%), it is critical to assess the patient's dynamic balance and mobility following the stroke [14]. The vestibular rehabilitation therapy (VRT) includes visual stabilization exercises (i.e., eye movements training), voluntary head movements (i.e., management of the vestibulo-ocular reflex VOR), and training balance control with decreasing of base of support using various positions of the head and trunk while performing different upper limbs tasks (i.e., enhancing of vestibulospinal regulation and improving awareness to avoid falls) $[15,16]$.

VRT techniques were used as an effective modality in managing balance disturbance in patients who had suffered a concussion as a consequence of traumatic brain injury. VRT improves balance (i.e., dynamic and static) through utilizing specific exercises targeting the patients' specific problems [17]. VRT has been recommended as a complementary intervention for balance improvement in patients with moderate impairments who have Progressive Supranuclear Palsy (PSP) during gait rehabilitation [18].

The current study aims to evaluate the effect of VRT in hemineglect rehabilitation and determine whether the improvement is sustained following training. It is hypothesized that patients receiving the VRT program will show more significant improvement in spatio-temporal gait parameters than patients receiving only the traditional physiotherapy program.

\section{Materials and methods}

The study followed a randomized controlled trial. The study was performed between May 2019 and April 2020 and registered on the clinicaltrials.gov website with identification number: NCT03913663. All patients were informed about the study's purpose, nature, and potential risks, and all participants signed a consent form indicating their agreement to participate in the present research study.

The research related to human use has complied with all the relevant national regulations, and institutional policies have followed the Declaration of Helsinki's tenets. The authors' institutional review board has approved them by the Ethical Committee of faculty of medicine, Beni-Suef University, with approval number FMBSUREC/05032019/EL-Hamrawy.

Participants: The study recruited 32 patients from Tanta University Hospital and Beni-Suef University Hospital. All patients were with left post-stroke hemineglect.A confrontation visual field exam was performed to exclude any visual field defects. All the patients received a physiotherapy program in the acute stage and post-discharge from the hospital. None of the patients enrolled in this study received a previous VRT program.

\section{Inclusion criteria}

Patients were included if they were $\geq 65$ years old, had a stroke within the preceding six months, and were able to walk independently. The presence of 


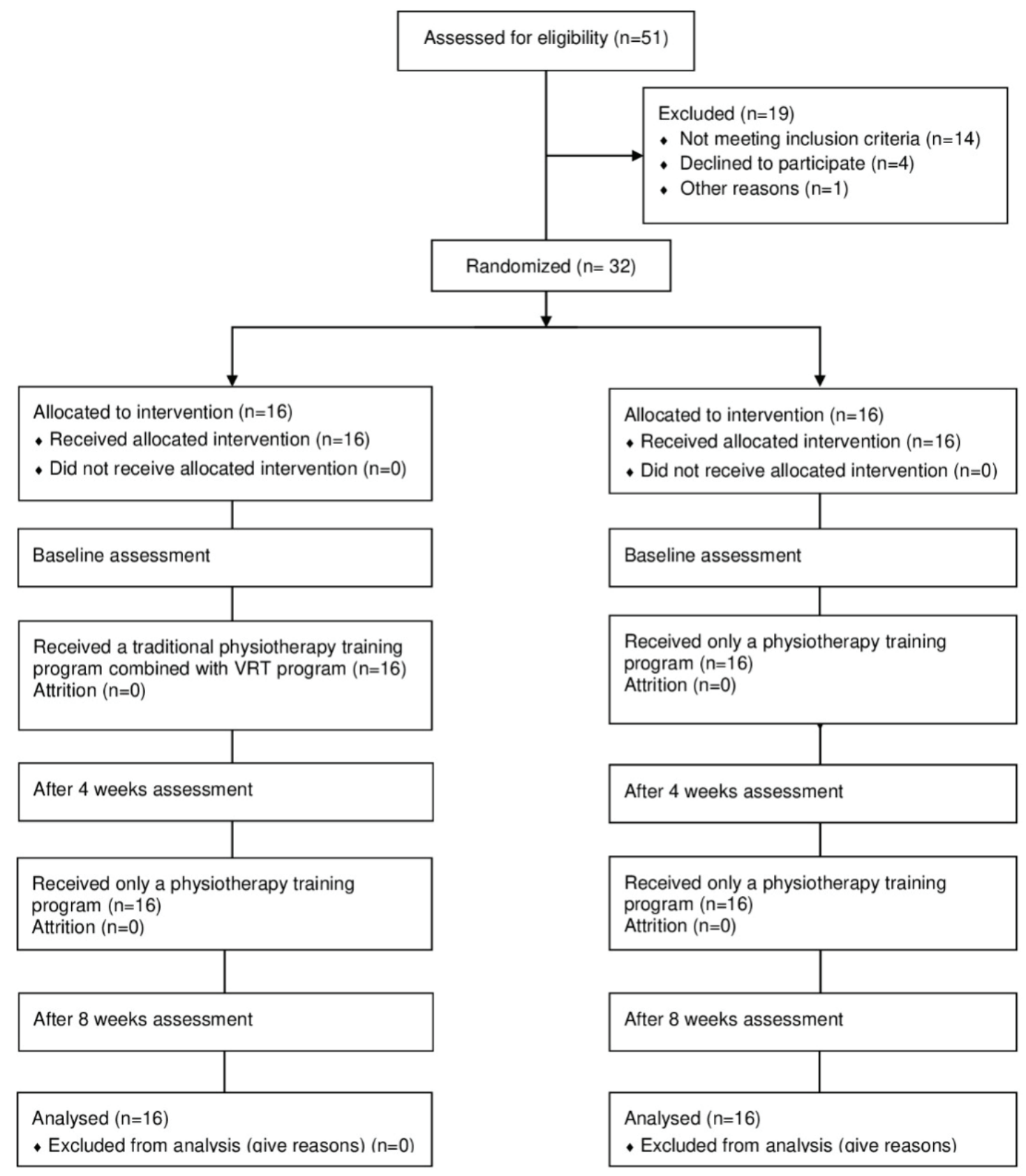

Fig. 1. The patients' flowchart

a first-time right hemispheric stroke that damaged the cerebral cortex, particularly the temporoparietal lobe (verified by computed tomography or MRI report), neurological examination, medical chart, and Star Cancellation Test.

Exclusion criteriaPatients who met one of the following criteria were excluded from the study: those with neurological or orthopedic problems or any severe comorbidity that affect gait, those who used walking aids or splints, those who were unable to provide informed consent for study participation, and those with only one functional eye.

\section{Intervention}

Each patient received a thorough explanation of the study's objectives, as well as a demonstration of equipment and procedures. The experimental group received a 60-minute training session consisting of traditional physiotherapy for 40 minutes and VRT for 20 minutes four days a week for the first four weeks, and then received a 60-minute session consisting solely of traditional physiotherapy for four weeks. For eight weeks, the control group followed only the traditional program for 60 minutes per day, four days per week. The traditional gait training program included strengthening 
exercises, walking over obstacles, up and down slopes, and 15:20 minutes of treadmill walking at a speed of $1.2-2.6 \mathrm{~km} / \mathrm{h}[19]$.

Each VRT session included exercises to improve eye movements and exercise improving postural control [15]:

- Eye movements: upward and downward, and on both sides, focusing on a finger that moves from $3 \mathrm{ft}$ to $1 \mathrm{ft}$ away from face (at first slowly, then quickly).

- Head movement: backward and forward bending, turning from right to left.

- Balancing on a mat and swiveling eyes.

- Standing on a sports mat, walking on the spot, and rotating the head on both sides.

- Alternately shift weight toward one limb while maintaining partial weight bearing on the other, keeping the sight on a fixed target object. Repeated for the opposite side.

- Standing on foam with closed eyes and rotating the head on both sides.

- Forward and backward walking with a side-to-side head turn.

- Sitting on a ball with feet on foam, eyes closed and bouncing slightly while turning the head from side to side.

- Marching in place

All patients in the experimental and control groups had their spatio-temporal gait parameters evaluated at baseline, four and eight weeks after the intervention. Spatio-temporal gait parameters were identified by using a markless 3D motion analysis sensor, the Microsoft Kinect V2 [20]. Each patient was asked to walk toward the Kinect sensor (Microsoft Kinect ${ }^{\mathrm{TM}}$ V2) maintaining a comfortable speed without being instructed to correct their gait. Patients were asked to wear fitting and non-reflective clothes to limit the tracking errors. The sensor was positioned on a tripod at a height of 0.8 $\mathrm{m}, 60$ degrees above the surface, and 4.5 meters from the starting line. The Kinect was connected to a laptop, and the data were analyzed using custom software [21]. The sampling rate of the Kinect was 15 frames/sec during the measurements. The suggested walking track was 1.5 to $4.5 \mathrm{~min}$ in front of the sensor as illustrated in Fig. 2. Because the minimum operating distance for the camera is $0.5 \mathrm{~m}$ in front of it, we placed it further away than recommended to ensure accurate recording of at least one complete gait cycle [22]. Spatiotemporal variables, including speed, cadence, paretic step length, non-paretic step length and, step width, were calculated from the extracted joints' positions from the Microsoft Kinect [23]. The step length was described as the distance between the two feet in the walking direction. The step width was described as the lateral distance between the feet, at the time of a foot placement. The speed was calculated by dividing the sum of all step lengths by the sum of all step times within each recording [24].

\section{Data analysis}

The data were statistically processed using IBM SPSS Statistics version 25.0 for Windows (IBM Corp., Armonk, NY). Training-related effects were assessed by two-way analyses of variance (ANOVA) with repeated measures, between groups, and for each group pre and post- 4 weeks and 8 weeks of intervention.A post-hoc test with Bonferroni procedure was used in multiple comparisons both between and within groups. The effect sizes (Cohen's d) were calculated using the mean differences of the changes after 4 and 8 weeks compared with the baseline. The level of significance was set at 0.05 .

\section{Results}

The baseline characteristics of the patients were shown in Table 1. After baseline assessment, 16 patients were randomly assigned to the experimental group and

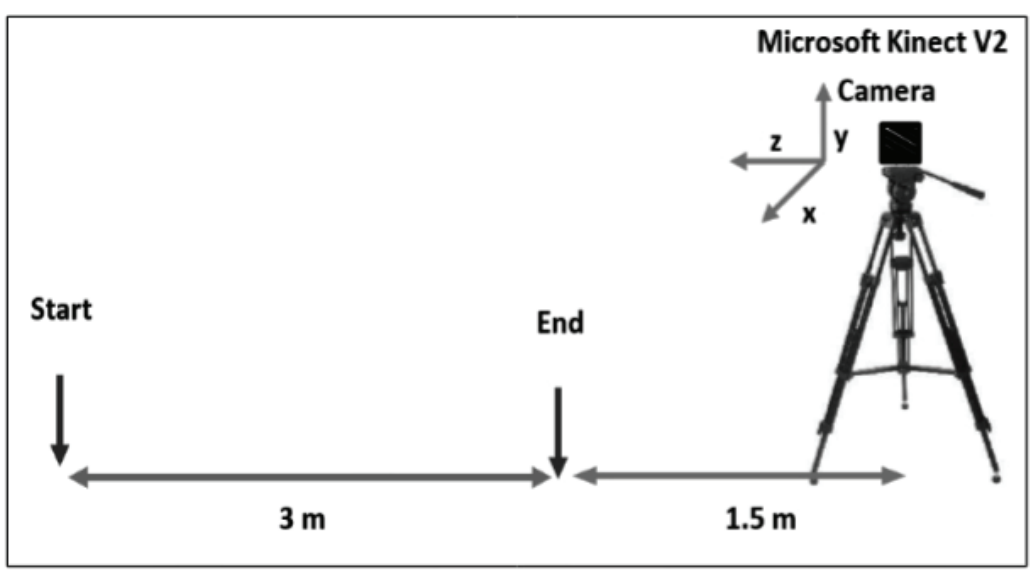

Fig. 2. The walking track 
Tab. 1. Baseline characteristics of the patients

\begin{tabular}{lcc}
\hline Variables & Experimental group & Control group \\
\hline Age $[$ mean $\pm \mathrm{SD}]$ & $66.5 \pm 3.15$ & $68.5 \pm 3.8$ \\
$\mathrm{Sex}, \mathrm{n}[\%$ Male] & $11[68.75]$ & $10[62.5]$ \\
$\mathrm{BMI}\left[\mathrm{kg} / \mathrm{m}^{2}\right]$ & $28.7 \pm 1.09$ & $28.07 \pm 0.95$ \\
Time post-stroke [months] & $8.81 \pm 1.55$ & $8.67 \pm 1.36$ \\
Ischemic/hemorrhagic & $12 / 4$ & $11 / 5$ \\
DGI score [mean $\pm \mathrm{SD}]$ & $11.9 \pm 1.38$ & $11.6 \pm 1.4$ \\
\hline
\end{tabular}

BMI: Body Mass Index, N: Number, SD: Standard Deviation

16 patients were assigned to the control group as shown in (Fig. 1). There were no significant differences at baseline between the experimental and the control groups in age, sex, BMI, months post-stroke, causes of the lesion, or dynamic gait index (DGI) scores.

Tables 2 and 3 show the changes in spatio-temporal gait parameters between the experimental and control groups. The post hoc analysis revealed that there were no significant differences between the groups at baseline for all spatio-temporal gait parameters. After training the experimental group showed that the speed increased significantly after 4 and 8 weeks with values of $\mathrm{P}=0.0001, \mathrm{~d}=12.38$, and $\mathrm{P}=0.001, \mathrm{~d}=13.69$, respectively, compared to the baseline. The cadence increased considerably after 4 and 8 weeks with values of
$\mathrm{P}=0.0003, \mathrm{~d}=3.88$, and $\mathrm{P}=0.0003, \mathrm{~d}=5.19$, respectively, compared to the baseline. The paretic step length increased significantly after 4 and 8 weeks compared with the baseline with values of $\mathrm{p}=0.0001, \mathrm{~d}=2.53$, and $\mathrm{p}=0.001, \mathrm{~d}=3.84$, respectively. The non-paretic step length increased significantly after 4 and 8 weeks compared with the baseline $\mathrm{p}=0.0119, \mathrm{~d}=2.06$, and $\mathrm{p}=0.0044, \mathrm{~d}=2.31$, respectively. The step width showed no significant differences in all the treatment period.

There were no significant differences in any of the spatio-temporal gait parameters in the control group. Cohen's d was calculated as post-training mean minus baseline mean divided by collected SD at baseline and post training.

Tab. 2. Changes in spatio-temporal gait parameters in the experimental group $(n=16)$

\begin{tabular}{lccccccc}
\hline \multirow{2}{*}{ Parameter } & \multirow{2}{*}{ Baseline } & \multirow{2}{*}{ 4 weeks } & \multirow{2}{*}{8 weeks } & \multicolumn{2}{c}{ Baseline vs. 4 weeks } & \multicolumn{2}{c}{ Baseline vs. 8 weeks } \\
\cline { 6 - 8 } & & & & $\mathrm{p}$ & $\mathrm{d}$ & $\mathrm{d}$ \\
\hline Speed [m/sec] & $0.39 \pm 5.6$ & $0.52 \pm 6.9$ & $0.53 \pm 7.2$ & 0.0002 & 12.38 & 0.001 & 13.69 \\
Cadence [steps/min] & $63.75 \pm 4.0$ & $67.63 \pm 3.2$ & $68.9 \pm 3.9$ & 0.0003 & 3.88 & 0.0003 & 5.19 \\
Paretic step length [cm] & $28.81 \pm 1.8$ & $31.3 \pm 1.3$ & $32.8 \pm 1.1$ & 0.0001 & 2.53 & 0.001 & 3.84 \\
Non-paretic step length [cm] & $26.06 \pm 1.9$ & $28.13 \pm 2.2$ & $28.4 \pm 2.6$ & 0.0119 & 2.06 & 0.0044 & 2.31 \\
Step width [cm] & $22.09 \pm 1.8$ & $22.3 \pm 1.79$ & $22.5 \pm 1.6$ & 0.9777 & 0.13 & 0.7887 & 0.41 \\
\hline
\end{tabular}

Tab. 3. Changes in spatio-temporal gait parameters in the control group $(n=16)$

\begin{tabular}{lccccccc}
\hline \multirow{2}{*}{ Parameter } & \multirow{2}{*}{ Baseline } & \multirow{2}{*}{ 4 weeks } & 8 weeks & \multicolumn{2}{c}{ Baseline vs. 4 weeks } & \multicolumn{3}{c}{ Baseline vs. 8 weeks } \\
\cline { 6 - 8 } & & & $\mathrm{p}$ & $\mathrm{d}$ & $\mathrm{p}$ & $\mathrm{d}$ \\
\hline Speed [m/sec] & $0.38 \pm 7.42$ & $0.40 \pm 7.8$ & $0.41 \pm 7.1$ & 0.9657 & 0.7 & 0.7323 & 1.88 \\
Cadence [steps/min] & $64.27 \pm 3.0$ & $64.9 \pm 2.9$ & $65.1 \pm 4.46$ & 0.4295 & 1.69 & 0.8750 & 0.8 \\
Paretic step length [cm] & $29.26 \pm 1.7$ & $29.8 \pm 1.6$ & $30.01 \pm 2.0$ & 0.6140 & 0.63 & 0.5261 & 0.72 \\
Non-paretic step length [cm] & $26.56 \pm 1.8$ & $27.01 \pm 2.1$ & $27.37 \pm 2.3$ & 0.1538 & 1.3 & 0.7144 & 0.57 \\
Step width [cm] & $21.3 \pm 1.9$ & $21.2 \pm 2.43$ & $21.2 \pm 2.07$ & 0.9332 & 0.3 & 0.9618 & 0.19 \\
\hline
\end{tabular}




\section{Discussion}

The results of this study indicated that the experimental group improved significantly in spatio - temporal gait parameters, such as speed, cadence, paretic and non-paretic step length, but the step width showed non-significant changes. It is worth noting that this improvement was maintained after training, and there was an additional improvement in gait characteristics compared to baseline.Patients with hemineglect exhibit severe postural instability as a result of both intra-body stabilization and spatial orientation problems. It may be assumed that these problems are caused by a partial loss of visual feedback from the affected side serving to control posture and the neglected phenomenon [25].

About $50 \%$ of human steps during walking and ADLs activities are redirected. Patients post-stroke have difficulty in redirecting movement because of defects of the motor system that provides information on the head movement and different body positions [26]. The exercise-based vestibular rehabilitation involved eyehead coordination exercises, postural control, balance training, and gaze stabilization training. The extraocular efferent motion perception and afferents (proprioception) promote visual stabilization and significantly improve postural control [9].

VRT demonstrated a statistically significant improvement in spatio - temporal gait parameters due to an increase in body shifting and symmetrical weight-bearing with changes in head movement, as described in the VRT exercises [27,28]. In the present study, various oculomotor exercises were manipulated while varying body positions, which may result in an improvement in patients' postural stability and spatial orientation during walking $[29,30]$.

Training hemineglect patients with closed or open eyes on different positions and surfaces may improve the patients' heading control while walking toward a target and their sustained attention and alertness over longer periods [31,32]. Different eye movements with regards to head movements maintain sight focused on a target are manipulated by vestibular and visual inputs. Thus, training eye movements relative to the target are used to orient body position, explore infer in space, and control upright posture by improving recognition of self-motion with the surrounding environment in the visual field $[33,34]$.In terms of training balance in poststroke survivors, it has been established that eye movement exercises resulted in major changes in walking speed, cadence, and step length over time, as well as significant differences following training [35].

According to a previous study, VRT may improve the vestibulo-ocular reflex function and gait control in patients with vestibular disease and stroke hemiparesis
[36]. It has been reported that eye movement training in post-stroke survivors brought about significant changes in walking speed, cadence, and step length over time, resulting in significant differences following training [37].

A previous study discovered a significant improvement in relative walking speed during navigation in a controlled environment resembling the real world in patients with retinitis pigmentosa (RP), who have severe peripheral visual field loss impairing mobility, as well as the maintenance of the improvement after training [38]. In our study, the improvement of spatio - temporal gait parameters might be attributed to improving the navigation and exploration of the hemiblind space in patients during walking.

\section{Conclusions}

The vestibular rehabilitation therapy effectively improves the spatio - temporal gait parameters (speed, cadence, paretic and non-paretic step length) in patients with post-stroke hemineglect, allowing patients to walk more securely and navigate the hemiblind space easily during walking. VRT might improve the patients' postural control, weight distribution, and orientation during walking. This improvement was maintained after training, and an additional improvement of spatio temporal gait parameters were observed compared to baseline. VRT is important in the rehabilitation of gait in hemineglect.

\section{Funding}

This research received no external funding

\section{Conflict of interest}

The authors have no conflict of interest to declare.

\section{References}

1. Thomas JO, Barrett AM. Right brain stroke syndromes. In: Stroke Rehabilitation. Elsevier; 2019. p. 71-89.

2. Cubelli R. Definition: Spatial neglect. Cortex. 2017; 92: 320-1.

3. Caggiano P, Jehkonen M. The "neglected" personal neglect. Neuropsychol Rev. 2018; 28(4): 417-35.

4. Li K, Malhotra PA. Spatial neglect. Pract Neurol. 2015; 15(5): 333-9.

5. Vanbellingen T, Ottiger B, Maaijwee N, Pflugshaupt T, Bohlhalter S, et al. Spatial neglect predicts upper limb use in the activities of daily living. Cerebrovasc Dis. 2017; 44(3-4): 122-7.

6. Doron N, Rand D. Is unilateral spatial neglect associated with motor recovery of the affected upper extremity 
poststroke? A systematic review. Neurorehabil Neural Repair. 2019; 33(3): 179-87.

7. Kutlay S, Genç A, Gök H, Öztuna D, Küçükdeveci A. Kinaesthetic ability training improves unilateral neglect and functional outcome in patients with stroke: A randomized control trial. J Rehabil Med. 2018; 50(2): 159-64.

8. Ogourtsova T, Archambault PS, Lamontagne A. Poststroke unilateral spatial neglect: virtual reality-based navigation and detection tasks reveal lateralized and non-lateralized deficits in tasks of varying perceptual and cognitive demands. J NeuroengRehabil. 2018; 15(1): 34 .

9. Fischetti F, Cataldi S, Giunto A, Greco G. Effect of home-based oculomotor exercises on postural stability in healthy female adults. J Hum Sport Exerc. 2019; 15(3): 653-60.

10. Moon Y, Sung J, An R, Hernandez ME, Sosnoff JJ. Gait variability in people with neurological disorders: A systematic review and meta-analysis. Hum Mov Sci. 2016; 47: 197-208.

11. Tian S, Li M, Wang Y, Chen X. Application of an Improved Correlation Method in Electrostatic Gait Recognition of Hemiparetic Patients. Sensors. 2019; 19(11): 2529.

12. Lee HH, Jung SH. Prediction of post-stroke falls by quantitative assessment of balance. Ann Rehabil Med. 2017; 41(3): 339-46.

13. Pirker W, Katzenschlager R. Gait disorders in adults and the elderly: A clinical guide: A clinical guide. Wien KlinWochenschr. 2017; 129(3-4): 81-95.

14. Kim O, Kim J-H. Falls and use of assistive devices in stroke patients with hemiparesis: Association with balance ability and fall efficacy. RehabilNurs. 2015; 40(4): 267-74.

15. Han BI, Song HS, Kim JS. Vestibular rehabilitation therapy: review of indications, mechanisms, and key exercises. J Clin Neurol. 2011; 7(4): 184-96.

16. Brandt T, Dieterich M. Postural imbalance in peripheral and central vestibular disorders. In: Bronstein AM, Brandt T, Woollacott MH, Nutt JG. Clinical Disorders of Balance, Posture and Gait. 2nd ed. London:Arnold, 2004; 146-62.

17. Gurley JM, Hujsak BD, Kelly JL. Vestibular rehabilitation following mild traumatic brain injury. NeuroRehabilitation. 2013; 32(3): 519-28.

18. Zampieri C, Di Fabio RP. Improvement of gaze control after balance and eye movement training in patients with progressive supranuclear palsy: a quasirandomized controlled trial. Arch Phys Med Rehabil. 2009; 90(2): 263-70.

19. Chang WH, Kim MS, Huh JP, Lee PK, Kim YH. Effects of robot-assisted gait training on cardiopulmonary fitness in subacute stroke patients: a randomized controlled study. Neurorehabil Neural Repair 2012; 26: 318-24.

20. Guffanti D, Brunete A, Hernando M, Rueda J, Navarro Cabello E. The accuracy of the Microsoft Kinect V2 sensor for human gait analysis. A different approach for comparison with the ground truth. Sensors. 2020; 20(16): 4405.

21. Elkholy A, Hussein M, Gomaa W, Damen D, Saba E. Efficient and Robust Skeleton-Based Quality Assessment and Abnormality Detection in Human Action Performance. IEEE J Biomed Health Inform. 2020; 24(1): 280-91.

22. Noonan P, Howard J, Hallett W, Gunn R. Repurposing the Microsoft Kinect for Windows v2 for external head motion tracking for brain PET. Phys Med Biol. 2015;60(22):8753-66.

23. Mentiplay B, Perraton L, Bower K et al. Gait assessment using the Microsoft Xbox One Kinect: Concurrent validity and inter-day reliability of spatiotemporal and kinematic variables. J Biomech. 2015; 48(10): 2166-70.

24. Müller B, IlgW, Giese MA, Ludolph N. Validation of enhanced kinect sensor based motion capturing for gait assessment. PLOS ONE. 2017; 12(4): e0175813.

25. Pérennou D. Postural disorders and spatial neglect in stroke patients: a strong association. Restor Neurol Neurosci. 2006; 24(4-6): 319-34.

26. Segal AD, Orendurff MS, Czerniecki JM, Shofer JB, Klute GK. Local dynamic stability in turning and straight-line gait. J Biomech. 2008; 41(7): 1486-93.

27. Park C-S, Kang K-Y. The effects of additional action observational training for functional electrical stimulation treatment on weight bearing, stability and gait velocity of hemiplegic patients. J Phys Ther Sci. 2013; 25(9): 1173-5.

28. Lee S-B, Kang K-Y. The effects of isokinetic eccentric resistance exercise for the hip joint on functional gait of stroke patients. J Phys Ther Sci. 2013; 25(9): 1177-9.

29. Vereeck L, Wuyts F, Truijen S, De Valck C, Van de Heyning P. The effect of early customized vestibular rehabilitation on balance after acoustic neuroma resection. Clin Rehabil. 2008; 22(8): 698-713.

30. Schubert M, Della Santina C, Shelhamer M. Incremental angular vestibulo-ocular reflex adaptation to active head rotation. Exp Brain Res. 2008; 191(4): 435-46.

31. Ogourtsova T, Archambault PS, Lamontagne A. Poststroke visual neglect affects goal-directed locomotion in different perceptuo-cognitive conditions and on a wide visual spectrum. Restor Neurol Neurosci. 2018; 36(3): 313-31.

32. García-Muñoz C, Cortés-Vega M-D, Heredia-Rizo AM, Martín-Valero R, García-Bernal M-I, CasusoHolgado MJ. Effectiveness of vestibular training for balance and dizziness rehabilitation in people with 
multiple sclerosis: A systematic review and meta-analysis. J Clin Med. 2020; 9(2): 590.

33. Rodrigues S, Polastri P, Carvalho J, Barela J, Moraes R, Barbieri F. Saccadic and smooth pursuit eye movements attenuate postural sway similarly. Neurosci Lett. 2015; 584: 292-5.

34. Guerraz M, Bronstein A. Ocular versus extraocular control of posture and equilibrium. Neurophysiologie Clinique/Clinical Neurophysiology. 2008; 38(6): 391-8.

35. Elhamrawy M, Saif MY, Mohamed S, Said M. Effect of eye movement training on balance in post stroke patients with unilateral spatial neglect. First preliminary report. NILES JGeriatrGerontol. 2019; 2(1): 71-6.
36. Mitsutake T, Sakamoto M, Ueta K, Oka S, Horikawa E. Effects of vestibular rehabilitation on gait performance in poststroke patients: a pilot randomized controlled trial. Int J Rehab Res. 2017; 40(3): 240-5.

37. Kang K, Yu K. The effects of eye movement training on gait function in patients with stroke. J PhysTher Sci. 2016; 28(6): 1816-8.

38. Ivanov I, Mackeben M, Vollmer A, Martus P, Nguyen N, Trauzettel-Klosinski S. Eye Movement Training and Suggested Gaze Strategies in Tunnel Vision A Randomized and Controlled Pilot Study. PLoS One. 2016; 11(6): e0157825. 